\section{LA COMPETITIVIDAD: UN ELEMENTO CLAVE EN LA EMPLEABILIDAD}

\author{
Pedro Manuel Aguado Benedí \\ Departamento Matemática Aplicada, Escuela de Ingeniería y \\ Arquitectura. Universidad de Zaragoza \\ Gerente de la Fundación Empresa-Universidad de Zaragoza \\ Presidente de la Red de Fundaciones Universidad-Empresa
}

\begin{abstract}
The article attempts to describe a plan that aims to promote the employability of graduates by conducting a training period in a company, being accompanied in the process of developing through a series of training and reinforcement adequate mentoring, as well as boost their entrepreneurial capacity.
\end{abstract}

KEY WORDS: Employability; training; entrepreneurial capacity.

\section{LA FORMACIÓN Y EL EMPLEO: LA EMPLEABILIDAD}

La mundialización de los intercambios, la globalización de las tecnologías y en particular la sociedad de la información, han aumentado las posibilidades de acceso de los individuos a la información y el conocimiento.

Al mismo tiempo todos estos fenómenos, conllevan una modificación de las competencias adquiridas y de los sistemas de trabajo.

Se ve claramente que las nuevas posibilidades ofrecidas a los individuos, exigen un esfuerzo de adaptación para construirse uno mismo su propia cualificación, recomponiendo los conocimientos ya adquiridos y adquiriendo otros nuevos, a través de procedimientos flexibles, actuales y eficaces.

Por otra parte la sociedad de la información, tenderá a cambiar el funcionamiento de la empresa que ofrecerá nuevos horizontes a la educación y la formación, lo cual supondrá nuevos retos que habrá que afrontar, algunas de las cuales son:

\section{COMPETITIVENESS: A KEY ELEMENT IN EMPLOYABILITY}

RESUMEN: El artículo trata de describir un plan que tiene como objetivo favorecer la inserción laboral de los titulados mediante la realización de una estancia formativa en una empresa, estando acompañado en el proceso de desarrollo de esa estancia, a través de una serie de refuerzos formativos y una tutoría adecuada, a la vez que impulsar su capacidad emprendedora.

PALABRAS CLAVE: Empleabilidad; prácticas; capacidad emprendedora.

- Facilitar, al máximo, la movilidad de los estudiantes y titulados, fomentando el reconocimiento mutuo de los créditos de enseñanza, sobre todo en el ámbito de la Unión Europea.

- Trabajar intensamente en la eliminación de los obstáculos administrativos, jurídicos y de protección social que actualmente frenan este tipo de intercambios.

- Fomentar la constitución de empresas y la creación de mecanismos que favorezcan la creación de microempresas, por parte de los jóvenes.

De la misma forma que hoy nadie discute que la formación profesional es un elemento clave para las políticas activas de empleo, hay que asumir que lo mismo ocurre con la formación continua a la hora de configurar lo que podriamos llamar políticas o estrategias activas de la competitividad empresarial.

Si la formación inicial es un instrumento eficaz para acceder al mercado laboral, la formación continua es, en sus aspectos de actualización y reciclaje, el medio más adecuado para el mantenimiento del empleo y la promoción dentro del mismo. 
En lo que se refiere al aspecto más difuso como son las aptitudes sociales que se demandan, se puede concretar que los rasgos más comúnmente solicitados son los referentes a las capacidades relacionales, al comportamiento en el trabajo y a toda una gama de competencias que corresponden al nivel de responsabilidad ocupado y que posteriormente serán considerados desde el punto de vista de perfiles solicitados

Por ello, en este contexto, será necesario:

- Establecer redes de cooperación, entre instituciones de investigación, empresas y centros de enseñanza, en general.

- Mejorar el acceso a la educación y la formación, así como su calidad.

- Fomentar el potencial humano dedicado a la investigación tanto pura como aplicada.

- Incrementar la competitividad y prevenir el desempleo, mediante la adaptación de la mano de obra al desafío del cambio a través de un enfoque sistemático de la formación continua.

Para lograr estos objetivos, las líneas de acción a seguir serán:

- Anticipación de las tendencias del mercado de trabajo y de las necesidades de formación.

- Reconversión, orientación, asesoramiento y formación especifica.

- Mejora y desarrollo de sistemas de formación adecuados y flexibles.

- Desarrollo de la planificación, promoción del establecimiento de redes de información interdisciplinares.

- Difusión y sensibilización de las innovaciones establecidas.

Será pues, fundamental que una vez estudiado el estado del arte del empleo tratemos de crear, incentivar y en definitiva ejecutar mecanismos e instrumentos que tiendan a dar soluciones a los problemas planteados en nuestra sociedad actual, en lo que se ha dado en Ilamar la empleabilidad.

Se pasa seguidamente a exponer lo que podría ser un ejemplo de actuación para poder lograr, cuando menos, parte de los objetivos mencionados y que supone un modelo real, basado en una metodología probada ya con buenos resultados y enriquecida, en este modelo, con aspectos puntuales considerados como importantes y fundamentales, como son el acompañamiento en el proceso de formación y el impulso al emprendimiento.

\section{Un Modelo de actuación}

El ejemplo presentado se denomina PRINCIPIA que responde a siglas obtenidas del nombre completo del programa y que describe el objetivo pretendido: Programa de Inserción a la Empresa y al ejercicio profesional y que tiene como herramienta básica, el desarrollo de prácticas o estancias en empresas, avalada por estudios y análisis realizados en la Fundación Empresa-Universidad de Zaragoza-FEUZ, desde hace varios años y de los que se deduce que la fórmula mencionada es muy bien acogida por los actores de la misma, los egresados/as por una parte y las empresas por la otra.

Algunos resultados obtenidos es exponen en la siguiente tabla (Tabla 1).

El programa tiene como principal objetivo:

Mejorar la empleabilidad de los titulados y tituladas universitarios/as y facilitar su contratación, así como la puesta en marcha de iniciativas emprendedoras que logren complementar, que no sustituir ni competir con aspectos o acciones que otro tipo de políticas de empleo puedan contemplar.

Dicho programa debería ser promovido, informando a las empresas de su existencia y promocionando a los/las participantes en el programa, a la vez que informar sobre las ayudas destinadas al fomento del empleo y asesorándoles en su tramitación.

Se trata por tanto de tener como objetivo facilitar el acceso al mercado laboral de los/las titulados/das, de acuerdo con los estudios realizados.

Con este objetivo el programa presentado contempla diferentes actividades como las prácticas formativas en empresas nacionales e internacionales, fomento del diálogo entre empleadores y potenciales empleados a través de la organización de encuentros, seminarios y 
TABLA 1: RESULTADOS DE BUENAS PRÁCTICAS EN LA FUNDACIÓN EMPRESA UNIVERSIDAD DE ZARAGOZA-FEUZ

\begin{tabular}{|c|c|c|c|c|c|}
\hline Movilidad Internacional & 2007 & 2008 & 2009 & 2010 & $\%$ Total \\
\hline ¿La preparación lingüistica te permitió hacer frente a situaciones cotidianas?... & 71,63 & 62 & 74,6 & 64,29 & 68,12 \\
\hline ¿La preparación lingüistica te ayudó a trabajar en tu campo profesional?... & 68,27 & 62 & 70,2 & 60,71 & 65,29 \\
\hline ¿La organización de la estancia ha satisfecho tus necesidades profesionales?... & 81,25 & 85 & 80,3 & 78,57 & 81,27 \\
\hline ¿Has sabido claramente lo que se esperaba hicieras y aprendieras en tu estancia?... & 78,37 & 75 & 76,3 & 85,71 & 78,85 \\
\hline ¿La duración de la estancia ha sido suficiente?... & 61,54 & 73 & 70,2 & 71,43 & 69,038 \\
\hline ¿Las actividades realizadas estaban directamente relacionadas con tu formación?... & 76,44 & 80 & 81,1 & 82,14 & 79,93 \\
\hline ¿Tuviste el equipamiento adecuado?... & 81,25 & 86 & 84,2 & 85,71 & 84,293 \\
\hline ¿Colaboraste con frecuencia con el tutor/ra encargado de la evaluación?... & 88,94 & 88 & 89,5 & 85,71 & 88,03 \\
\hline SATISFACCIÓN GLOBAL DE LA ESTANCIA Buena/Excelente & 84,13 & 86 & 87,7 & 92,86 & 87,678 \\
\hline He conocido nuevos métodos o técnicas & 80,29 & 81 & 79,4 & 82,14 & 80,705 \\
\hline He adquirido nuevas habilidades profesionales & 85,58 & 83 & 86 & 89,29 & 85,958 \\
\hline He mejorado el idioma & 88,94 & 82 & 92,1 & 89,29 & 88,085 \\
\hline Tengo más confianza en mi mismo/ma & 83,17 & 79 & 84,7 & 89,29 & 84,028 \\
\hline Entiendo mejor a otras personas & 80,77 & 78 & 84,2 & 85,71 & 82,173 \\
\hline Mi estancia me ayudará a encontrar trabajo & 87,02 & 82 & 86,8 & 89,29 & 86,288 \\
\hline SATISFACCIÓN GLOBAL SOBRE RESULTADOS Buena/Excelente & 85,1 & 90 & 89 & 92,86 & 89,25 \\
\hline \multicolumn{6}{|l|}{ Movilidad Nacional } \\
\hline ¿Las actividades realizadas estaban directamente relacionadas con tu formación?... & 79,84 & 77,8 & 85,9 & 78,5 & 80,5 \\
\hline ¿Colaboraste con frecuencia con el tutor/ra encargado de la evaluación?... & 63,51 & 73 & 76,5 & 71,96 & 71,24 \\
\hline ¿La organización de la la estancia ha satisfecho tus necesidades profesionales?... & 89,15 & 95,2 & 89,4 & 88,79 & 90,648 \\
\hline ¿Has sabido claramente lo que se esperaba hicieras y aprendieras en tu estancia?... & 77,52 & 77,8 & 81,2 & 77,57 & 78,513 \\
\hline SATISFACCIÓN GLOBAL DE LA ESTANCIA Buena/Excelente & 84,5 & 84,1 & 85,9 & 83,18 & 84,423 \\
\hline He adquirido nuevas habilidades profesionales & 89,06 & 94,8 & 92,6 & 91,71 & 92,053 \\
\hline Tengo más confianza en mi mismo/ma & 79,94 & 88,2 & 90,2 & 85,99 & 86,058 \\
\hline Entiendo mejor a otras personas & 78,42 & 74,8 & 79,8 & 81,16 & 78,553 \\
\hline ¿Has encontrado trabajo tras la práctica?... & 66,67 & 33,3 & 36,5 & 36,45 & 43,23 \\
\hline ¿En la misma empresa de la práctica?... & 64,37 & 71,4 & 67,7 & 76,92 & 70,115 \\
\hline
\end{tabular}

presentaciones en colaboración con los distintos Centros educativos, además de visitas personales en las sedes de las empresas.

Se realizarán estudios, análisis y mediciones, utilizando plataformas y herramientas adecuadas, en relación con las capacidades y competencias profesionales que tanto se demandan actualmente.

La base de estos modelos es la idea de que la inteligencia emocional conlleva la expresión de un determinado comportamiento y que la posesión de un elevado nivel de inteligencia emocional promueve competencias específicas que diferencian a los trabajadores y trabajadoras en el desarrollo de su tarea laboral.

Será pues fundamental la medición de las competencias mencionadas, de una manera fiable y adecuada y con una metodología debidamente contrastada.

En el presente programa se propone dicha medición a través de la utilización de una plataforma virtual basada

ARBOR Vol. 187 Extra 3 diciembre [2011] 15-21 ISSN: 0210-1963 
en modelos avanzados de dirección de personal y gestión de competencias, sustentado en un profundo conocimiento científico ampliamente experimentado en el entorno empresarial.

El programa de formación tiene como objetivo favorecer la inserción laboral de los titulados mediante la realización de una estancia formativa en una empresa, pero estando acompañado en el proceso de desarrollo de esa estancia, a través de una serie de refuerzos formativos y una tutoría adecuada.

La meta será la mejora de las competencias y habilidades profesionales del recién titulado además de complementar con formación práctica y experiencia la formación obtenida en la enseñanza reglada cursada.

Con este fin se elabora un plan diversificado en función de las necesidades del participante en el programa, el grado de competencias medido y el tipo de empresa en el que se vayan a desarrollar sus prácticas y consiste en un programa formativo, para recién titulados sin experiencia, en un ámbito funcional cualificado, con una tutorización efectiva y unos objetivos de formación práctica evaluable.

Dicha formación lectiva se efectuará a través de la utilización de herramientas informáticas contrastadas y sesiones presenciales interactivas dirigidas e impartidas por profesionales de experiencia en estos temas y formas de proceder que darán las claves que permitan un mejor aprovechamiento de la estancia formativa en la empresa.

Dentro del proceso de acompañamiento y mejora que supone este programa se incluye una evaluación de competencias que permite obtener una fotografía de cómo una persona gestiona la expresión de sus emociones y sus habilidades sociales en su vida cotidiana.

Se debe estar preparado para ser capaces de satisfacer a un cliente cada vez más exigente y donde las unidades de trabajo surgen (y desaparecen) en función del mercado y requieren ejecutivos con mentalidad empresarial. Tener talentos preparados para ese desafío constituye una diferencia estratégica de magnitud.
En este contexto la persona deberá pensar con el siguiente esquema mental:

Debo ser emprendedor/ra, en un ámplio sentido del concepto pero... ¿Qué es y qué implica un desarrollo en la capacidad emprendedora?

1. Un cambio de actitud, lo cual es fundamental puesto que ahora la persona no vale por lo que sabe sino por lo que hace con lo que sabe.

2. Aprender la forma de agregar valor permanentemente a la organización. Las empresas compran ahora el resultado del tiempo, no el tiempo. Por lo tanto, si no se agrega valor, no vale.

3. Implica ser empleable, atractivo con talento para el mercado laboral y, por consiguiente, muy cotizado para crecer dentro de la empresa.

4. Transformarse de espectador de los cambios dentro de la empresa a protagonista de los mismos.

5. Fijarse metas propias y tomar la iniciativa.

6. Establecer normas personales de calidad altas que repercutan en un resultado para la empresa.

7. Estar dispuestos a superar los errores y fracasos, como experiencias necesarias.

8. Saber administrar bien el riesgo.

9. Generar lealtad hacia los objetivos a largo plazo.

10. Trabajar en equipo y saber reconocer a sus colaboradores.

Con este planteamiento y al objeto de cumplir los resultados marcados, se deberá elaborar una metodología apropiada que incluya tanto los contenidos a desarrollar, como la dinámica para realizarlo y que podría resumirse en el siguiente gráfico (Gráfico 1).

Toda esta metodología da como consecuencia que aquél que la practica y la aprovecha, adquiere una serie de competencias transversales que da como resultado una mayor competitividad de cara al mercado laboral y de cara a los potenciales empleadores.

\section{La Competitividad}

Es el momento de incidir en este concepto tan manejado en la actualidad y que tantas connotaciones tiene y será procedente recordar que una definición del concepto podría expresarse, de acuerdo con el diccionario, como: 

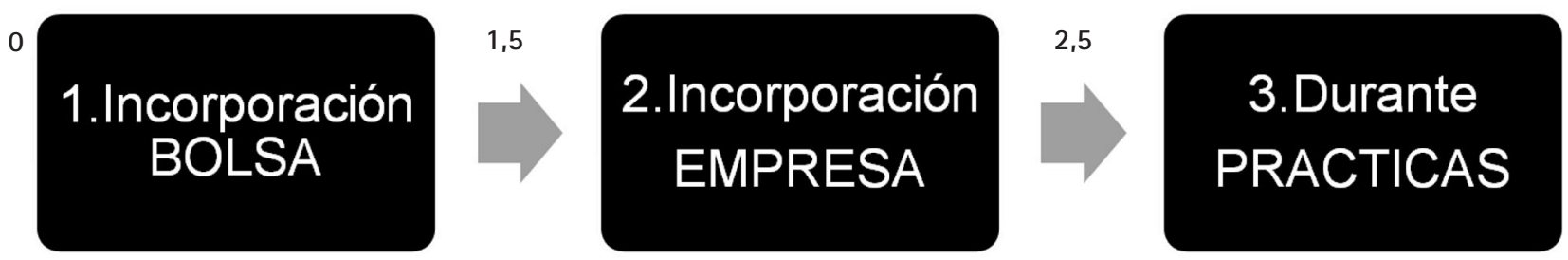

\section{SESIÓN FORMATIVA. EMPRESA Y HABILIDADES INTRA EMPRENDEDORAS}

1.5. Píldoras formativas online

\section{SESIÓN COACHING PRESENCIAL incorporación empresarial}

2. 5. Herramientas formativas online

\section{COACHING ONLINE durante prácticas}

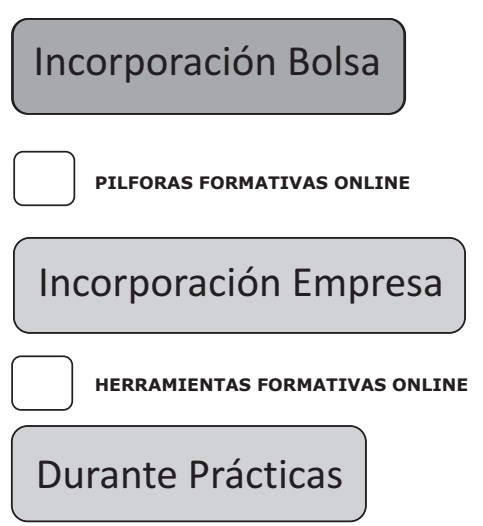

Destinatarios.

1.000 participantes

2/4 sesiones mensuales

Máx. 20 alumnos/sesión

SESIÓN FORMATIVA

"EMPRESA Y HABILIDADES INTRAEMPRENDEDORAS»

\section{Objetivos}

- Detectar y Activar Talento.

Contenido

- Empresa. Un ser vivo.

- Mi Proyecto y Dafo profesional.

Iniciativa, autoconfianza, automotivación.

- Práctica habilidades. Empatía, Gestión del

Tiempo, Creatividad y adaptación al cambio,

Inteligencia emocional, Iniciativa, liderazgo,

Responsabilidad, Habilidad comercial,

Resolución de problemas, Negociación,

Trabajo en equipo.

Duración: 8 horas

Gráfico 1

Competencia intensa. Calidad de una acción o de un producto, por la cual éstos pueden establecer competencia con otros de su clase en términos de igualdad.

La competitividad en el contexto de la empleabilidad debe entenderse como la adquisición y manejo de cualidades que suponen una diferenciación con el resto de aspirantes a entrar en el mercado laboral. En este sentido, las competencias transversales, tan demandadas en los perfiles buscados, constituyen elementos diferenciadores y por otra parte necesarias para introducirse con ciertas garantías en el mercado del trabajo. Es por ello que esta metodolo- 
gía de las prácticas deben ser cuidadas, perfeccionadas y evaluadas para que den los frutos apetecidos y buscados, como son la preparación efectiva del universitario/a, tras su periodo de adquisición de conocimientos en la carrera académica reglada cursada.

Al objeto de reforzar la importancia que las prácticas formativas tienen, se puede hacer referencia al estudio efectuado en el presente año por la Red de Fundaciones Universidad Empresa, Red FUE, que agrupa a 29 Fundaciones que representan a 40 Universidades y en el cual se ve que, de acuerdo con el Gráfico 2, la actividad mencionada representa casi un $40 \%$ de su actividad total.

Fondos Gestionados por Áreas de Actividad Año 2010

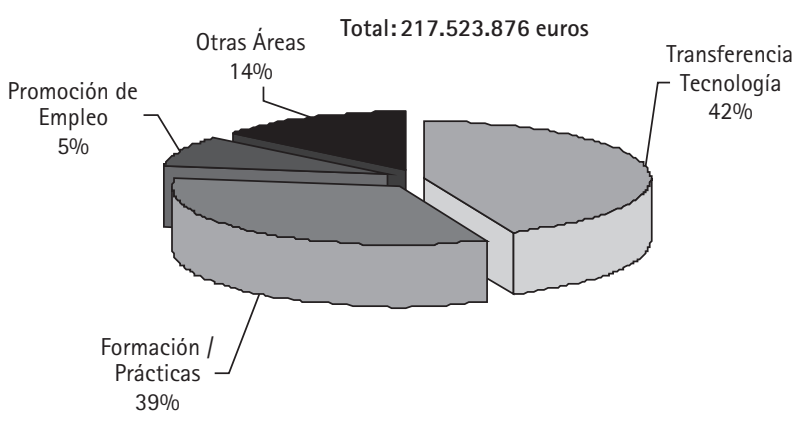

Gráfico 2

Este tipo de formación práctica se lleva a efecto, por ahora, de forma sucesiva a su carrera académica, pero debería llegar a realizarse de forma simultánea a ésta, en períodos perfectamente predefinidos de antemano.

Esta forma de proceder daría como consecuencia el logro de una demanda que el mundo empresarial está solicitando ya, la llamada "formación dual", entendida ésta como una formación integral tanto en el campo teórico como en el práctico y tanto en el terreno nacional como internacional y todo ello realizado de forma conjunta por la "Academia": la universidad y el "Mercado": las empresas.

En el contexto mencionado se integra pues el programa Principia expuesto y tampoco deben olvidarse otras fórmulas muy empleadas por los universitarios/as y que tienen muy buena acogida entre los futuros empleadores, como son las Becas Erasmus y las Becas Leonardo, que pueden ser complementarias o coadyuvantes al plan mencionado.

Todo lo expuesto hasta ahora, deberá traducirse en una mejora de la capacidad de inserción laboral del egresado/ da, pues debería contribuir a complementar los distintos aspectos que son requeridos en los perfiles solicitados y que podrían resumirse en lo que se denominaria "perfil estándar" solicitado por la empresa, que se podria traducir en la necesidad de poseer: -Conocimiento del entorno económico y empresarial, -Valores personales, -Formación "dual": teórica y práctica, nacional e internacional, -Idiomas: inglés, alemán, -Movilidad nacional e internacional, -Experiencia internacional: Erasmus, -Disposición de aprender: cambio, -Mentalidad intercultural, -Trabajo en equipo, Capacidades analíticas... análisis/sintesis, -Orientación a resultados.

Lo cual interpretado de forma resumida se podría expresar como: universitario/a que ha finalizado sus estudios académicos en el tiempo adecuado, curso por año o cercano a ello, con un adecuado nivel de los conocimientos correspondientes, una práctica probada de éstos y con los complementos de unas competencias de carácter transversal, así como del dominio del idioma inglés y conocimientos de otro.

Para terminar, se cita el estudio realizado por Infoempleo, año 2011 y donde algunas de las conclusiones obtenidas ,y de forma extractada, son que la necesidad de demostrar que se tiene cierta experiencia está presente en el 76,3\% de las ofertas.

El inglés es el más solicitado (72\%) y la lengua francesa es requerida para un $8,5 \%$ de los puestos, con un creciente interés por otras lenguas como el chino, el japonés 0 el árabe, y una mayor demanda de idiomas de paises de Europa del Este.

Un $46 \%$ de las ofertas de empleo cualificado indica nivel o titulación universitaria especifica, donde las titulaciones técnicas son el $42 \%$, las de tipo jurídico-social el 32,4\%, las de ámbito científico y sanitario un $20,6 \%$ y las de humanidades un $4,7 \%$. 
Para 2012 Infoempleo.com establece tres escenarios: crecimiento anual del PIB del 1,2\%, o del 0,7\% o del 0,3\%, en todos los casos el sector servicios lidera la generación de empleo, seguido a una distancia considerable por el sector industrial.
El ayudar a la consecución de estos objetivos, en su mayor y mejor grado y desde el punto de vista de formadores y docentes debe constituir también nuestro objetivo y nuestra meta.

\section{BIBLIOGRAFÍA}

Aguado, P. (2011): Los servicios universitarios de empleo en el contexto del EEES. Ponencia. www.ual.es

AVD Asesores (2011): Programa Intraemprendedores. Manual, 17 pp.
Cámara Zaragoza (2011): Desarrollar competencias: Aprender a aprender. Manual, $41 \mathrm{pp}$.

Infoempleo.com. (2011): Informe Oferta y demanda de empleo en España, 238 pp.

Red FUE (2011): Informe Encuesta red de fundaciones universidad-empresa, 20 pp. 